\title{
Comparison of Multi-Channel Ranging Algorithms for Narrowband LPWA Network Localization
}

\author{
Florian Wolf ${ }^{1,2}$, Mohamed Sana ${ }^{1}$, Sébastien de Rivaz ${ }^{1}$, François Dehmas ${ }^{1}$ and \\ Jean-Pierre Cances ${ }^{2}$ \\ ${ }^{1}$ CEA-Leti Minatec Campus, 17 rue des Martyrs, 38054 Grenoble Cedex 09, France \\ Email: \{florian.wolf, mohamed.sana, sebastien.derivaz, francois.dehmas\}@cea.fr \\ ${ }^{2}$ Université de Limoges, CNRS, XLIM, UMR 7252, 87000 Limoges, France \\ Email: \{florian.wolf, jean-pierre.cances\}@xlim.fr
}

\begin{abstract}
Accurate radio signal based localization for Low Power Wide Area networks enables ubiquitous positioning for the Internet of Things. Narrowband communication and multipath propagation make precise localization challenging. Coherent multi-channel ranging increases bandwidth and provides improved temporal resolution through the aggregation of sequentially transmitted narrowband signals. This paper applies parametric estimators as well as a deep learning technique to multi-channel measurements obtained with $10 \mathrm{kHz}$ signals. Ranging performances are compared via numerical simulations and real outdoor field trials, where parametric estimation and deep learning achieve $60 \mathrm{~m}$ and $45 \mathrm{~m}$ accuracy in $90 \%$ of the cases, respectively. Further work is required to study the impact of deep neural network training with a combination of synthetic and real data. Future research may also include the adaptation of multi-channel localization to differential network topologies.
\end{abstract}

Keywords: LPWA network localization, range estimation, frequency hopping, MUSIC, deep learning, DNN

\section{Introduction}

Narrowband Low Power Wide Area (LPWA) networks based on radio technologies such as LoRa, Sigfox and NB-IoT provide wireless connectivity to low data rate devices in the framework of the Internet of Things (IoT) [1].

The localization of these devices enables new applications such as object tracking, allows adding context information to device-generated data or can be used for enhanced network management [2]. While integrating a Global Navigation Satellite System (GNSS) module ensures meter-level positioning, certain use cases exclude this option due to power, form factor or cost constraints. Extracting location information from LPWA radio signals carrying the transmitted data addresses these issues. However, obtaining precise position estimates from low data rate and narrowband signals, required for long-range communication over several kilometers, remains challenging [3]. Low temporal resolution results in the difficulty to accurately estimate the Time-of-Arrival (ToA) of the direct path in multipath scenarios.

A brief literature review on LPWA network ranging and localization techniques is given in the following. In a mesh network, range estimates are obtained with a parametric signal path loss model and Received Signal Strength (RSS) measurements [4]. Due to blockage and small-scale fading, it is difficult to determine a valid model for RSS based ranging. To account for these shortcomings, RSS measurements combined with fingerprinting algorithms [5] achieve, once a robust database has been established, accuracy up to $500 \mathrm{~m}$. Precision of ToA based ranging techniques is inverse proportional to signal bandwidth [6]. LPWA networks implementing Time-Difference-of-Arrival (TDoA) techniques obtain location estimates through hyperbolic trilateration with a $500 \mathrm{~m}$ precision [7]. 


\section{2/12 F. Wolf et al.}

Coherent multi-channel ranging [6] aggregates multiple sequentially transmitted narrowband signals on different frequencies to form a virtual increased bandwidth. This technique, similar to stepped frequency radar, is compatible with LPWA transceivers and improves temporal resolution [8]. Various implementations [9-12] have demonstrated a higher accuracy over single-channel time based ranging techniques. However, these studies have focused on short-range technologies (i.e. WiFi, Zigbee, RDIF) and mostly on indoor propagation scenarios.

Multi-channel range estimation is based on a sampled version of the channel transfer function. The delay estimation problem can be addressed through MUltiple SIgnal Classification (MUSIC) [13], allowing an improved resolution of multipath over Fourier transformation based techniques [14, 15].

Recent advances in machine learning for wireless communications have opened up new horizons for the design of more flexible algorithms [16]. For range estimation problems, some of these algorithms are based upon Deep Neural Network (DNN) [17] or Convolutional Neural Network (CNN) [18] and are trained either using synthetically generated data or real-life measurements. The resulting solutions are shown to be competitive with respect to the state-of-art approaches while being flexible, as they comprise straightforward calculations [17].

The contributions of this paper, on coherent multi-channel ranging, are:

- Formulation of the multi-channel ranging signal model for the high-resolution algorithm MUltiple SIgnal Classification (MUSIC). Comparison of the direct channel impulse reconstruction by Inverse Discrete Fourier Transform (IDFT) and a MUSIC based range estimator in a two-path propagation scenario by numerical simulations.

- Design of a Deep Neural Network (DNN) based regression algorithm for range estimation, based on synthetically generated multi-channel transfer function data. Ranging performance comparison with IDFT and MUSIC by numerical simulations.

- Application and comparison of the parametric estimators (IDFT and MUSIC) as well as the deep learning algorithm (DNN) on real outdoor narrowband LPWA ranging field trials.

Range estimation through the IDFT technique i.e. as in [10] serves throughout this work as state of the art baseline approach in order to establish a fair comparison with MUSIC and DNN based ranging in the context of narrowband LPWA networks.

This paper is organized as follows. Section 2 introduces the multi-channel signal model and presents the range estimators. Comparison of the range estimation approaches is given by numerical simulation in Section 3. The multi-channel ranging transceiver testbed and the application of the range estimation techniques to real outdoor field trial measurements is discussed in Section 4. Section 5 concludes with perspectives.

\section{Coherent Multi-Channel Ranging}

\subsection{Signal model}

The basic transmission signal model including transmitter, radio channel and receiver is depicted in Fig. 1a. The energy normalized narrowband LPWA baseband signal $s_{0}$ is up-converted to transmit frequency $f_{0}$ resulting in the transmit signal

$$
s_{\mathrm{TX}}(t)=\mathcal{R}\left\{s_{0}\left(t^{[T]}(t)\right) e^{j\left(2 \pi f_{0} t^{[T]}(t)+\phi_{\mathrm{R}}^{[T]}\right)}\right\},
$$

where the transmitter local time $t^{[T]}(t)$ is function of global time $t$ applying to both baseband signal and up-conversion operation and $\mathcal{R}(\cdot)$ is the real part. After passing through the radio channel, the received signal in global time $t$ can be described by

$$
s_{\mathrm{RX}}(t)=s_{\mathrm{TX}}(t) * h(t),
$$




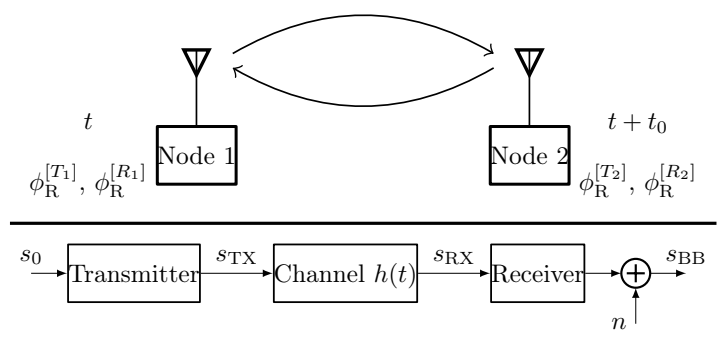

(a) Phase-of-Flight (PoF) measurement and transmission model.

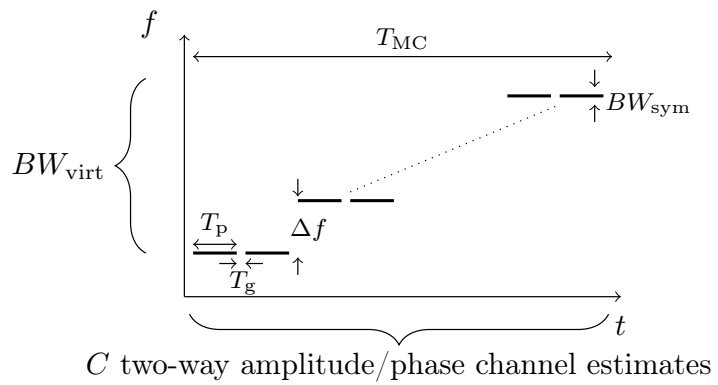

(b) Ranging protocol aggregating $C$ sequentially transmitted narrowband signals $\left(B W_{\text {sym }}\right)$ to a virtual bandwidth $B W_{\text {virt }}=(C-1) \Delta f$.

Fig. 1: Coherent multi-channel ranging.

with convolution operator $*$ and energy normalized channel impulse response, combining $P$ multipath components

$$
h(t)=\sum_{p=0}^{P-1} \alpha_{p} \delta\left(t-\tau_{p}\right) \quad \longrightarrow H(f)=\sum_{p=0}^{P-1} \alpha_{p} e^{-j 2 \pi f \tau_{p}},
$$

where $d=c_{0} \tau_{0}$ is the direct path and speed of light $c_{0}=3 \cdot 10^{8} \mathrm{~m} / \mathrm{s}$. After down-conversion, the low pass filtered received baseband signal is given by

$$
s_{\mathrm{BB}}(t)=s_{\mathrm{RX}}(t) e^{-j\left(2 \pi f_{0} t^{[R]}(t)+\phi_{\mathrm{R}}^{[R]}\right)}+n^{[R]}(t),
$$

where $t^{[R]}(t)$ is the receiver local time applying to the down-conversion operation only and Additive White Gaussian Noise (AWGN) $n$ of variance $\sigma^{2}=1 /\left(2 E_{\mathrm{s}} / N_{0}\right)$.

Combining (1), (2) and (4) results in the general received baseband signal

$$
s_{\mathrm{BB}}(t)=\left[s_{0}\left(t^{[T]}(t)\right) e^{j\left(2 \pi f_{0} t^{[T]}(t)+\phi_{\mathrm{R}}^{[T]}\right)} * h(t)\right] e^{-j\left(2 \pi f_{0} t^{[R]}(t)+\phi_{\mathrm{R}}^{[R]}\right)}+n^{[R]}(t) .
$$

In the following, a time offset $t_{0}$ between node 1 and node 2 and a zero Sampling/Carrier Frequency Offset (SFO/CFO) is assumed. Assigning node 1 as reference node, local to global time relations are expressed by

$$
\begin{aligned}
& t^{\left[T_{1}\right]}(t)=t^{\left[R_{1}\right]}(t)=t, \\
& t^{\left[T_{2}\right]}(t)=t^{\left[R_{2}\right]}(t)=t+t_{0} .
\end{aligned}
$$

For a transmission from node 1 to node 2, the resulting received signal according to (5) and (6) is given by

$$
s_{\mathrm{BB}}^{\left[T_{1}, R_{2}\right]}(t)=\left[s_{0}(t) e^{j\left(2 \pi f_{0} t+\phi_{\mathrm{R}}^{\left[T_{1}\right]}\right)} * h(t)\right] e^{-j\left(2 \pi f_{0}\left(t+t_{0}\right)+\phi_{\mathrm{R}}^{\left[R_{2}\right]}\right)}+n^{\left[R_{2}\right]}(t) .
$$

The frequency domain representation of (7), without noise $n$, can be written as

$$
S_{\mathrm{BB}}^{\left[T_{1}, R_{2}\right]}(f)=S_{0}(f) H\left(f+f_{0}\right) e^{-j 2 \pi f_{0} t_{0}} e^{j\left(\phi_{\mathrm{R}}^{\left[T_{1}\right]}-\phi_{\mathrm{R}}^{\left[R_{2}\right]}\right)} .
$$


Assuming normalization $S_{0}(f=0)=1$ and evaluating ${ }^{3}(8)$ at $f=0$, results in

$$
H_{c}^{\left[T_{1}, R_{2}\right]}=\left.\left(S_{\mathrm{BB}}^{\left[T_{1}, R_{2}\right]}(0)\right)\right|_{\left(f_{0}=f_{\mathrm{R}}+c \Delta f\right)}=H\left(f_{0}\right) e^{-j 2 \pi f_{0} t_{0}} e^{j\left(\phi_{\mathrm{R}}^{\left[T_{1}\right]}-\phi_{\mathrm{R}}^{\left[R_{2}\right]}\right)} .
$$

For time synchronized nodes i.e. $t_{0}=0$, a sampled estimation of the channel transfer function $H(f)$ can be obtained by performing narrowband transmissions from node 1 to node 2 according to Fig. $1 \mathrm{~b}$ at frequencies $f_{0}=f_{\mathrm{R}}+c \Delta f$ with $c \in[0 \ldots C-1]$. This one-way transmission, generally called channel estimation, is not sufficient for range estimation between unsynchronized nodes $\left(t_{0} \neq 0\right)$ as the delay $\tau$ of the radio channel, i.e. $h(t)=\alpha \delta(t-\tau)$ and $t_{0}$ are linearly dependent. A two-way exchange with a transmission from node 2 to node 1 results in $s^{\left[T_{2}, R_{1}\right]}$. Equivalent to (9), the frequency domain representation is given by

$$
H_{c}^{\left[T_{2}, R_{1}\right]}=H\left(f_{0}\right) e^{j 2 \pi f_{0} t_{0}} e^{j\left(\phi_{\mathrm{R}}^{\left[T_{2}\right]}-\phi_{\mathrm{R}}^{\left[R_{1}\right]}\right)} .
$$

Combining (9) and (10) eliminates the unknown time reference $t_{0}$

$$
\tilde{H}_{c}=H_{c}^{\left[T_{1}, R_{2}\right]} H_{c}^{\left[T_{2}, R_{1}\right]}=H^{2}\left(f_{\mathrm{R}}+c \Delta f\right) e^{j\left(\phi_{\mathrm{R}}^{\left[T_{1}\right]}-\phi_{\mathrm{R}}^{\left[R_{2}\right]}+\phi_{\mathrm{R}}^{\left[T_{2}\right]}-\phi_{\mathrm{R}}^{\left[R_{1}\right]}\right)} .
$$

This sampled version of the channel transfer function serves as input to the range estimators discussed in Section 2.2.

\subsection{Range estimators}

Three major issues need to be considered for range estimation based on (11):

- The sum-difference of local oscillator phases $\Delta \phi_{\mathrm{R}}=\phi_{\mathrm{R}}^{\left[T_{1}\right]}-\phi_{\mathrm{R}}^{\left[R_{2}\right]}+\phi_{\mathrm{R}}^{\left[T_{2}\right]}-\phi_{\mathrm{R}}^{\left[R_{1}\right]}$ needs to be constant over the set of frequencies $f_{\mathrm{R}}+c \Delta f$. This requirement can be achieved by an appropriate transceiver architecture, i.e. a common local oscillator (LO) for transmitter and receiver $\phi_{\mathrm{R}}^{\left[T_{x}\right]}=\phi_{\mathrm{R}}^{\left[R_{x}\right]}$ yielding $\Delta \phi_{\mathrm{R}}=0$ or numerical intermediate frequency mixing $c \Delta f$ for both transmitter $T_{x}$ and receiver $R_{x}$ and continuous operation of the radio frequency LO at $f_{\mathrm{R}}$ leading to $\Delta \phi_{\mathrm{R}}=$ const. [8].

- The combination of (9) and (10) in (11) to eliminate unknown time reference $t_{0}$, results in the square of the channel transfer function $H^{2}(f)$. Due to the $2 \pi$ ambiguity of phase measurements, recovering $H_{c}$ with the square-root operation introduces a $1 \pi$ ambiguity per channel. Techniques to estimate the resulting $2^{C}$-state error function under certain hypothesis and conditions are studied in [11]. For the general case, range estimation can be based on $\tilde{H}_{c}$, considering supplementary virtual paths in the convoluted channel impulse response $h * h$. For example, in a scenario with $P=2$, peaks will appear at $2 \tau_{0}, \tau_{0}+\tau_{1}$ and $2 \tau_{1}$ with amplitudes $\alpha_{0}^{2}, 2 \alpha_{0} \alpha_{1}$ and $\alpha_{2}^{2}$ respectively.

- For such a processing scheme, the $2 \pi$ ambiguity translates to a range ambiguity $R_{\max }=\frac{c_{0}}{2 \cdot \Delta f}$ and the virtual bandwidth $B W_{\text {virt }}=(C-1) \Delta f$ limits range resolution $\Delta R=\frac{c_{0}}{2 \cdot B W_{\text {virt }}}[6]$.

In the following, four range estimators based on (11) are studied.

Phase slope For a single propagation path $(P=1)$, the estimated channel transfer function (11) is given by $\tilde{H}_{c}=\alpha_{0}^{2} e^{-j 2 \pi c \Delta f 2 \tau_{0}}$. Range information can be extracted from the slope of the argument of $\tilde{H}_{c}$

$$
\hat{d}_{\text {slope }}=\tau c_{0}=-\frac{c_{0}}{4 \pi \Delta f} \frac{\Delta \arg \left(\tilde{H}_{c}\right)}{\Delta c} .
$$

\footnotetext{
${ }^{3}$ In practice, evaluation is performed by cross correlation with $s_{0}$ in time domain [8].
} 
Inverse Discrete Fourier Transform In multipath scenarios $(P>1)$, the reconstructed channel transfer function (11) can be converted to an estimate of the convoluted channel impulse response $h * h$ by Inverse Discrete Fourier Transform (IDFT)

$$
\tilde{h}_{k}=\operatorname{IDFT}\left\{\tilde{H}_{c}\right\} \text {. }
$$

In order to detect the first/direct path in multipath scenarios with possibly stronger secondary paths, the following first path detection scheme is applied. The range estimate $\hat{d}_{\text {IDFT }}$ is taken as the first local maximum above a certain threshold $\gamma$ relative to and in a certain range $R_{\text {first }}$ before the global maximum in the estimated channel impulse response $\tilde{h}_{k}$. Sufficient zero padding ensures fine time and hence range granularity. However, range resolution remains bound by the virtual bandwidth $B W_{\text {virt }}$.

MUltiple SIgnal Classification The estimation of amplitudes $\alpha_{p}$ and delays $\tau_{p}$ in the reconstructed channel transfer function can be formulated as a spectral estimation problem to which high-resolution techniques such as MUltiple SIgnal Classification (MUSIC) can be applied [13]. Therefore (11) with (3) is expanded and rewritten as

$$
\tilde{H}_{c}=\sum_{p=0}^{\tilde{P}-1} \tilde{\alpha}_{p} e^{-j 2 \pi\left(f_{\mathrm{R}}+c \Delta f\right) \tilde{\tau}_{p}},
$$

where $\tilde{P}, \tilde{\tau}_{p}$ and $\tilde{\alpha}_{p}$ account for the notation after expansion. Analog to [13], defining an imaging vector

$$
\phi^{C}\left(\tilde{\tau}_{p}\right)=\left[1 e^{-j 2 \pi \Delta f \tilde{\tau}_{p}} \ldots e^{-j 2 \pi(C-1) \Delta f \tilde{\tau}_{p}}\right]^{\mathrm{T}}
$$

and

$$
\boldsymbol{\Phi}^{C}=\left[\phi^{C}\left(\tilde{\tau}_{0}\right) \phi^{C}\left(\tilde{\tau}_{1}\right) \ldots \phi^{C}\left(\tilde{\tau}_{\tilde{P}-1}\right)\right],
$$

allows reformulating (14) in matrix form as

$$
\tilde{\boldsymbol{H}}=\left[\begin{array}{lll}
\tilde{H}_{0} & \ldots & \tilde{H}_{C-1}
\end{array}\right]^{\mathrm{T}}=\boldsymbol{\Phi}^{C} \tilde{\boldsymbol{\alpha}}+\tilde{\boldsymbol{N}}
$$

with amplitude $\tilde{\boldsymbol{\alpha}}=\left[\begin{array}{llll}\tilde{\alpha}_{0} & \tilde{\alpha}_{1} & \ldots & \tilde{\alpha}_{\tilde{P}-1}\end{array}\right]^{\mathrm{T}}$ and noise $\tilde{\boldsymbol{N}}$.

The construction of the Hankel matrix

$$
\boldsymbol{K}=\left(\begin{array}{cccc}
\tilde{H}_{0} & \tilde{H}_{1} & \cdots & \tilde{H}_{C-L-1} \\
\tilde{H}_{1} & \tilde{H}_{2} & \cdots & \tilde{H}_{C-L} \\
\vdots & \vdots & \vdots & \vdots \\
\tilde{H}_{L} & \tilde{H}_{L+1} & \cdots & \tilde{H}_{C-1}
\end{array}\right)
$$

with $1 \leq L<C$, allows the application of MUSIC to a single snapshot of the channel transfer function estimates $\tilde{\boldsymbol{H}}$ [13]. The singular value decomposition of the Hankel matrix (18) is given by

$$
\boldsymbol{K}=\left[\begin{array}{ll}
\boldsymbol{U}_{S} & \boldsymbol{U}_{N}
\end{array}\right] \operatorname{diag}\left(\lambda_{0} \lambda_{1} \ldots \lambda_{\tilde{P}-1} 0 \ldots 0\right)\left[\boldsymbol{V}_{S} \boldsymbol{V}_{N}\right]^{*},
$$

with singular values $\lambda_{0} \geq \lambda_{1} \geq \ldots \geq \lambda_{\tilde{P}-1} \geq 0$ and complex conjugate $(\cdot)^{*}$.

The set of delays $\tilde{\tau}_{p}$ can be obtained as the peaks in the imaging function $Y$, defined by the orthogonal projection of the imaging vector $\phi^{L}$ to the noise subspace $\boldsymbol{U}_{N}$

$$
Y(\tau)=\left\|\phi^{L}(\tau)\right\|_{2} /\left\|\boldsymbol{U}_{N}^{*} \phi^{L}(\tau)\right\|_{2} .
$$

The range estimate $\hat{d}_{\text {MUSIC }}$ is obtained by applying the first path detection to (20).

In contrast to the IDFT approach, delay estimation by the MUSIC algorithm can achieve arbitrary high resolution for sufficiently low noise [13]. 
Deep Neural Network based regression approach The problem considered so far can be viewed as determining a function $f^{\theta}: \mathbb{C}^{C} \rightarrow \mathbb{R}$ parameterized by $\theta$, which computes the range based on channel measurements. To find this function, thus the parameters $\theta$, a Deep Neural Network $(\mathrm{DNN})$ based regression algorithm is conducted on an expert database $\mathcal{D}=\left\{\left(\left\{\tilde{H}_{c, i}\right\}_{c=0}^{C-1} ; d_{i}\right)\right\}_{i=1}^{N}$, where $N=|\mathcal{D}|$ is the database size. Each entry $i$ in the database consists of a collection of channel measurements $\left\{\tilde{H}_{c, i}\right\}_{c=0}^{C-1}$ and the associated true range value $d_{i}$. During the training, the network parameters $\theta$ are updated via a gradient descend algorithm to minimize the loss function

$$
\mathcal{L}(\theta)=\mathbb{E}\left[\left(f^{\theta}\left(\left\{\tilde{H}_{c, i}\right\}_{c=0}^{C-1}\right)-d_{i}\right)^{2}\right] .
$$

During the exploitation phase, the range estimation $\hat{d}_{D N N}$ for a given channel measurement $\left\{\tilde{H}_{c}\right\}_{c=0}^{C-1}$ is obtained from the network parameter estimates $\hat{\theta}$ as $\hat{d}_{D N N}=f^{\hat{\theta}}\left(\left\{\tilde{H}_{c}\right\}_{c=0}^{C-1}\right)$.

\section{Numerical Simulations}

\subsection{Parametric estimators}

Numerical simulations are conducted to evaluate the performance of the presented range estimators on synthetic data. Simulations consider a two-path propagation scenario with a direct and a multipath component, where $\beta=\alpha_{1} / \alpha_{0}$ and $\Delta \tau=\tau_{1}-\tau_{0}$. The reconstructed channel transfer function estimates $\tilde{H}_{c}$ are obtained by (11) with $C=16$ and $\Delta f=200 \mathrm{kHz}$ leading to $B W_{\text {virt }}=3 \mathrm{MHz}$, $\Delta R=50 \mathrm{~m}$ and $R_{\max }=750 \mathrm{~m}$. AWGN of variance $\sigma^{2}$ is added to each channel estimate $H_{c}^{[T, R]}$. First path detection is performed on (13) and (20) with the optimized parameters $R_{\text {first }}=300 \mathrm{~m}$ and $\gamma=-7 \mathrm{~dB}$. MUSIC requires $P_{\text {MUSIC }}=\tilde{P}=3$ for two-path propagation. For each channel parameter configuration $\left(E_{\mathrm{s}} / N_{0}, \beta, \Delta \tau\right), 5000$ Monte Carlo simulations are performed.

Fig. 2a, Fig. 2c and Fig. 2e compare the maximum ranging error in $90 \%$ of the cases for the IDFT $\hat{d}_{\text {IDFT }}$ and MUSIC $\hat{d}_{\text {MUSIC }}$ estimator as function of the path delay difference $\Delta \tau$. For a path delay difference approximately equal to the multi-channel range resolution $(\Delta \tau \approx \Delta R)$, a maximum error is observed as overlapping main lobes form a larger lobe. The precision gain of MUSIC over the IDFT technique is most pronounced for path delay differences $\Delta \tau>100 \mathrm{~m}$ as MUSIC resolves multiple propagation paths and precision asymptotically attains single path precision. Range estimation degrades as the path amplitude ratio $\beta$ increases, which makes the detection of a weak first path difficult. For $\beta=10 \mathrm{~dB}$, first path detection does no longer find the first path below the threshold $\gamma$. Ranging errors then grow linearly with delay difference $\Delta \tau$. These findings are confirmed at high $E_{\mathrm{s}} / N_{0}$, where MUSIC clearly outperforms the IDFT approach as depicted in Fig. 2b, Fig. 2d and Fig. 2f.

\subsection{DNN based range estimation algorithm}

The database $\mathcal{D}$ is generated via Monte Carlo simulations. Table 1 summarizes the parameters used during simulations. Fig. 3 shows the DNN architecture, which comprises three hidden layers of 128 units. Each neuron has a Rectifier Linear Unit activation (ReLU) except the final layer. For a practical representation, each channel measurement is unpacked into real and imaginary part, thus, the input of the DNN is of size $2 C$. During the learning phase, a minibatch of 32 samples is randomly taken every iteration from the database on which the gradient descend is performed with an empirical step size or learning rate $\alpha=0.001$. Fig. 2 shows that the resulting DNN algorithm has comparable performance with respect to both IDFT and MUSIC algorithms. While for $\beta<0 \mathrm{~dB}$ it slightly performs less than the parametric methods, for $\beta \geq 0 \mathrm{~dB}$, the precision of the DNN algorithm takes over when $\Delta \tau<100 \mathrm{~m}$ 


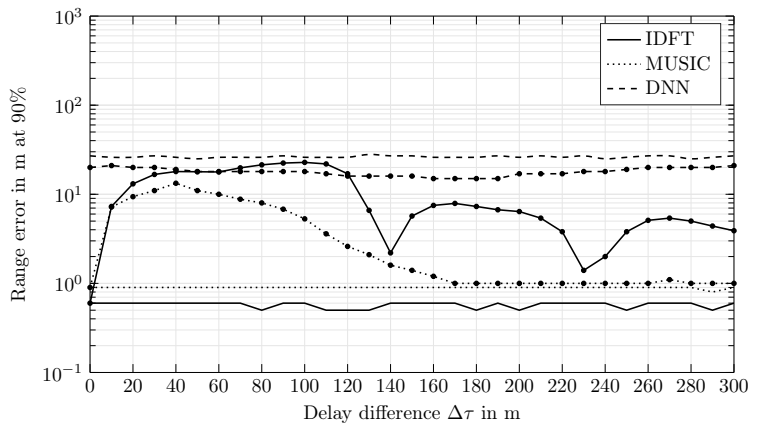

(a) $E_{\mathrm{s}} / N_{0}=25 \mathrm{~dB}, \beta=-30 \mathrm{~dB}(-),-3 \mathrm{~dB}(\bullet)$.

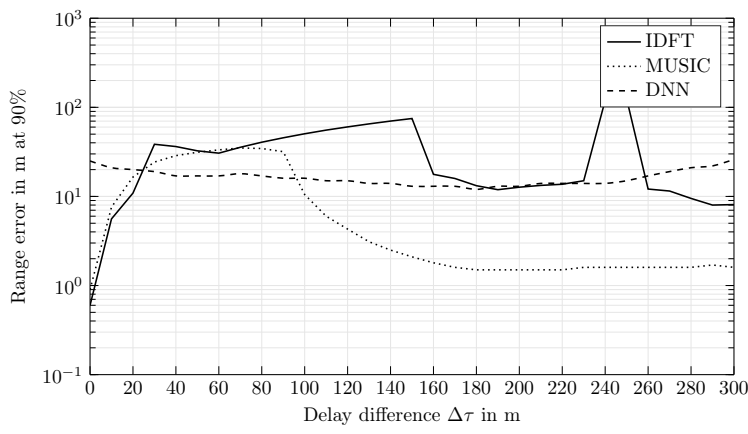

(c) $E_{\mathrm{s}} / N_{0}=25 \mathrm{~dB}, \beta=0 \mathrm{~dB}$.

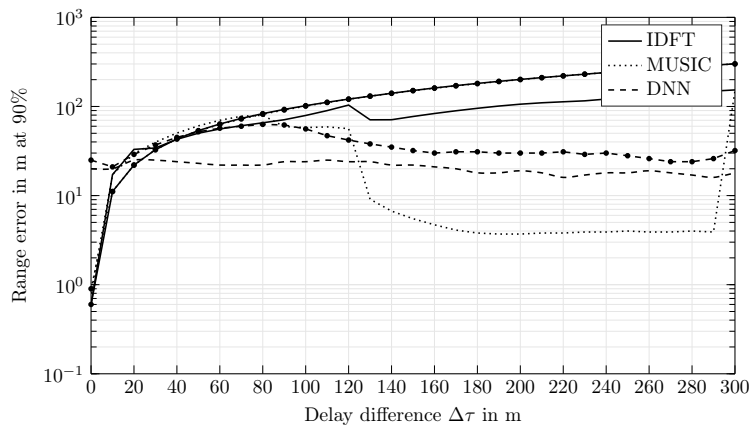

(e) $E_{\mathrm{s}} / N_{0}=25 \mathrm{~dB}, \beta=3 \mathrm{~dB}(-), 10 \mathrm{~dB}(\bullet)$.

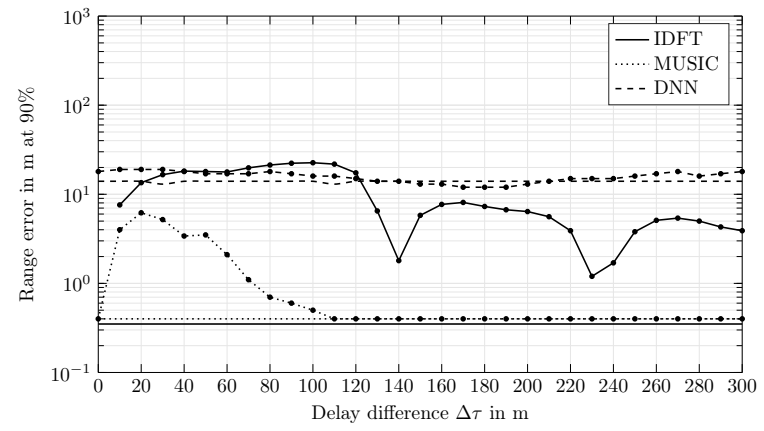

(b) $E_{\mathrm{s}} / N_{0}=50 \mathrm{~dB}, \beta=-30 \mathrm{~dB}(-),-3 \mathrm{~dB}(\bullet)$.

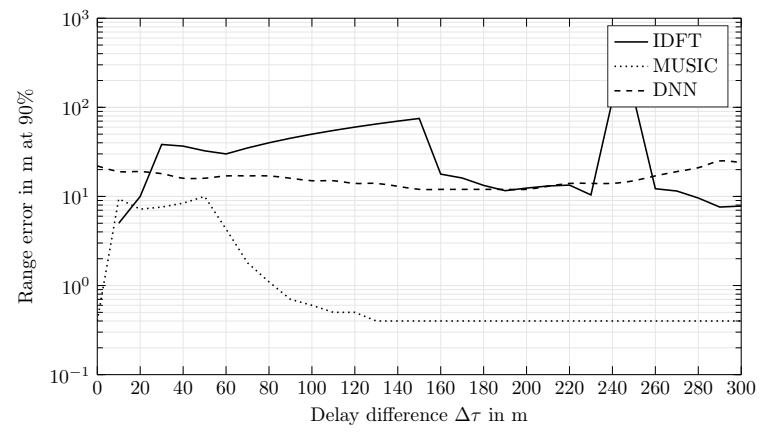

(d) $E_{\mathrm{s}} / N_{0}=50 \mathrm{~dB}, \beta=0 \mathrm{~dB}$.

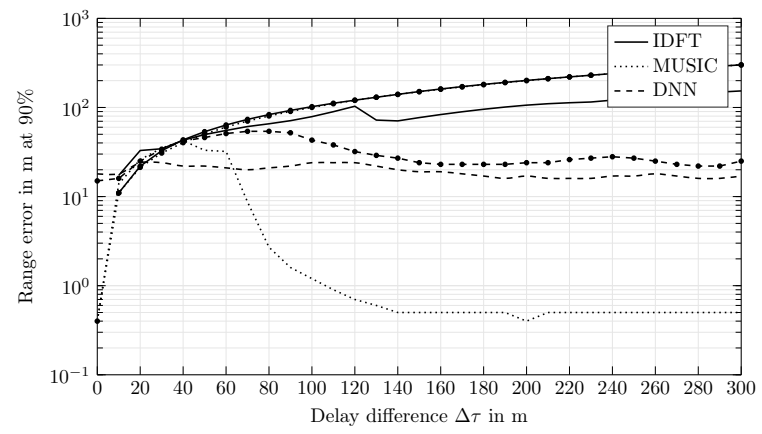

(f) $E_{\mathrm{s}} / N_{0}=50 \mathrm{~dB}, \beta=3 \mathrm{~dB}(-), 10 \mathrm{~dB}(\bullet)$.

Fig. 2: Simulated ranging error for IDFT, MUSIC and DNN estimators in a two-path propagation scenario.

and at low $E_{\mathrm{s}} / N_{0}$ but still remains lower than MUSIC technique when $\Delta \tau$ increases. In addition, it is worth to highlight that the proposed DNN algorithm is less sensitive to the variation of the parameters $\beta, \Delta \tau$, and $E_{\mathrm{s}} / N_{0}$. In fact, the range estimation error almost remains constant $(\approx 25 \mathrm{~m})$ when these parameters vary, demonstrating therefore the robustness of the DNN algorithm. 


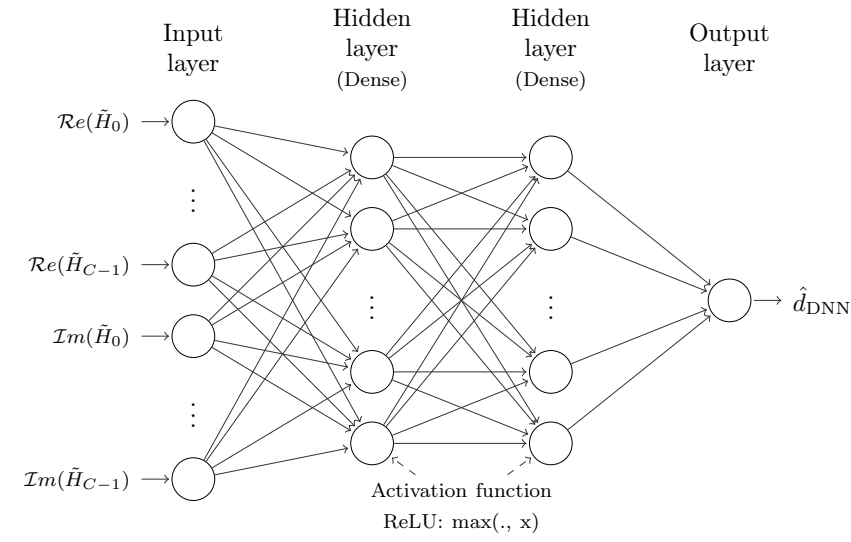

Fig. 3: Illustration of the DNN architecture for multi-channel range estimation.
Table 1: DNN training data generation parameters.

\begin{tabular}{l|c|r}
\hline Parameter & Symbol & Value \\
\hline \hline $\begin{array}{l}\text { Path } \\
\text { amplitude } \\
\text { ratio }\end{array}$ & $\beta=\alpha_{1} / \alpha_{0}$ & $\begin{array}{r}-30: 5:-5,-3,-1, \\
0,1,3,5,10 \mathrm{~dB}\end{array}$ \\
\hline $\begin{array}{l}\text { Path delay } \\
\text { difference }\end{array}$ & $\Delta \tau=\tau_{1}-\tau_{0}$ & $0: 10: 200 \mathrm{~m}$ \\
\hline $\begin{array}{l}\text { Signal-to- } \\
\text { noise ratio }\end{array}$ & $E_{\mathrm{s}} / N_{0}$ & $-30: 5: 50 \mathrm{~dB}$ \\
\hline $\begin{array}{l}\text { Monte } \\
\text { Carlo runs }\end{array}$ & & 1500 \\
\hline
\end{tabular}
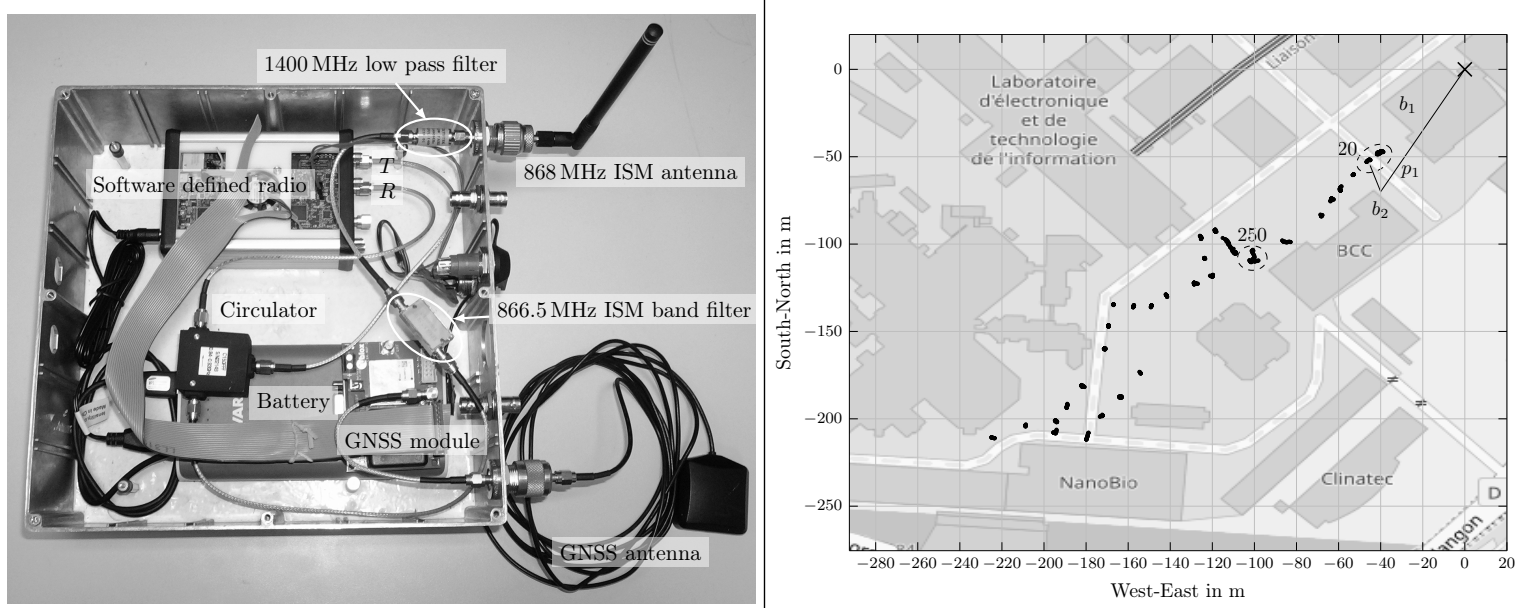

(a) Transceiver testbed comprising a SDR, radio (b) GNSS ground truth positions (.) w.r.t. the roof frequency components, a GNSS module and a power testbed $(\times)$ at $(0,0)$. supply.

Fig. 4: Multi-channel ranging field trials.

\section{Application to Field Trials}

\subsection{LPWA ranging transceiver testbed}

The transceiver testbed [8] illustrated in Fig. 4a comprises a Software Defined Radio ${ }^{4}$ (SDR), radio frequency components and a Global Navigation Satellite System (GNSS) module.

Digital intermediate frequency up-/down-mixing stages are implemented into the SDR, allowing to coherently process a $10 \mathrm{MHz}$ bandwidth by sequentially selecting $1 \mathrm{MHz}$ channels. Inphase/quadrature (IQ) samples are stored for offline processing by a dedicated software.

\footnotetext{
${ }^{4}$ Analog Devices AD9361 $2 \times 2$ TRX radio front end and a Zynq-045 Xilinx System on chip FPGA with
} integrated dual Cortex-A9 ARM processor. 
Two transceiver testbeds perform a multi-channel two-way ranging protocol according to Fig. 1, exchanging a Binary Phase Shift Keying (BPSK) preamble of chip rate $R_{\mathrm{c}} \equiv B W_{\text {sym }}=10 \mathrm{kHz}$ following a Gold code of chip length $N_{\mathrm{c}}=256$ in the $868 \mathrm{MHz}$ Industrial Scientific Medical (ISM) band. From $T_{\mathrm{g}}=3 \mathrm{~ms}$ and $T_{\mathrm{p}}=N_{\mathrm{c}} / B W_{\mathrm{sym}}=25.6 \mathrm{~ms}$ follows a total two-way multi-channel duration $T_{\mathrm{MC}}=2 C\left(T_{\mathrm{p}}+T_{\mathrm{g}}\right)=915.2 \mathrm{~ms}$.

The transceiver testbed comprises a uBlox GNSS module ${ }^{5}$ for the purpose of providing a ranging ground truth reference. In the following field trials, GNSS position estimates show a standard deviation $\sigma_{\text {GNSS }}<1 \mathrm{~m}$. Therefore, GNSS position errors are neglected and GNSS estimates are directly used to establish LPWA narrowband ranging errors.

\subsection{Outdoor field trial}

Outdoor field trials are performed between a node on the roof of a four-story building and a mobile node on the ground. GNSS reference positions for the measurements are depicted in Fig. 4b. A total of 900 valid measurements in stationary conditions is processed for range estimation. As indicated on the map in Fig. 4b, multipath propagation is possible due to surrounding buildings on the semi-urban industrial site.

\subsection{Ranging results and discussion}

In contrast to simulation, where $H_{c}^{[T, R]}$ are generated directly, field trial channel estimates $H_{c}^{[T, R]}$ for the construction of (11) are obtained by cross correlation of the received signals $s_{\mathrm{BB}, c}^{[T, R]}$ at the channel frequencies $c \Delta f$ with the transmit waveform $s_{0} H_{c}^{[T, R]}[8]$.

Fig. 5 shows the ranging errors for the different range estimation strategies. IDFT ranging precision in Fig. 5a is below $30 \mathrm{~m}$, however for certain positions large biases $(>100 \mathrm{~m})$ are observed. Processing the field trial measurements with the MUSIC algorithm in Fig. 5b does not decrease these biases, despite the high-resolution capacity. Possible causes are insufficient signal-to-noise ratio $E_{\mathrm{s}} / N_{0}$ (compare Fig. 2) or the mismatch between the hypothesis of a two-path channel $(\tilde{P}=3)$ and the real propagation channel.

Regarding the DNN approach, when trained on synthetic data and then applied on field trial data, the DNN algorithm (S-DNN) slightly performs less than the two parametric estimators (Fig. 5c). This is again because the hypothesis of a two-path channel is no longer valid in real environment, leading to channel measurements that were not seen in the synthetic database. The Cumulative Distribution Functions (CDF) in Fig. 6 summarize the ranging accuracy of the performed field trial.

In order to overcome the weakness of an incomplete database, training can be performed on a portion of the real field trial data (Fig. 5d). Fig. 7 shows the CDF (R-DNN) when trained on $2 / 3$ and applied to the remaining $1 / 3$ of the real field trial data in comparison to the performances when the synthetically-only trained DNN is applied to the same $1 / 3$ (S-DNN).

Furthermore, better results can be obtained by taking advantage of synthetic data, by using these data to pre-train the DNN and then refine it with some field trial data (M-DNN) [19], thus reducing the need of an extensive geo-referenced channel measurements database. Fig. 5e and Fig. 7 show the results for this approach. The resulting M-DNN has better performance than both S-DNN and R-DNN, therefore demonstrating the benefit of taking into consideration both real and synthetic data for a robust algorithm.

In conclusion, parametric estimators and synthetic data trained deep learning achieve a ranging error below $60 \mathrm{~m}$ to $80 \mathrm{~m}$ in $90 \%$ of the cases and mixed data trained deep learning attains $45 \mathrm{~m}$.

\footnotetext{
${ }^{5}$ uBlox C94-M8P application board.
} 
10/12 F. Wolf et al.

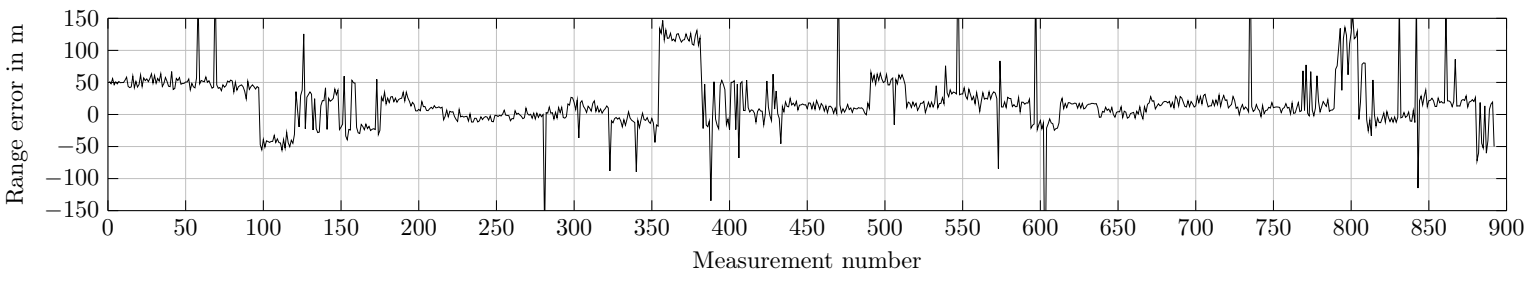

(a) Ranging error on IDFT $\hat{d}_{\text {IDFT }}$.

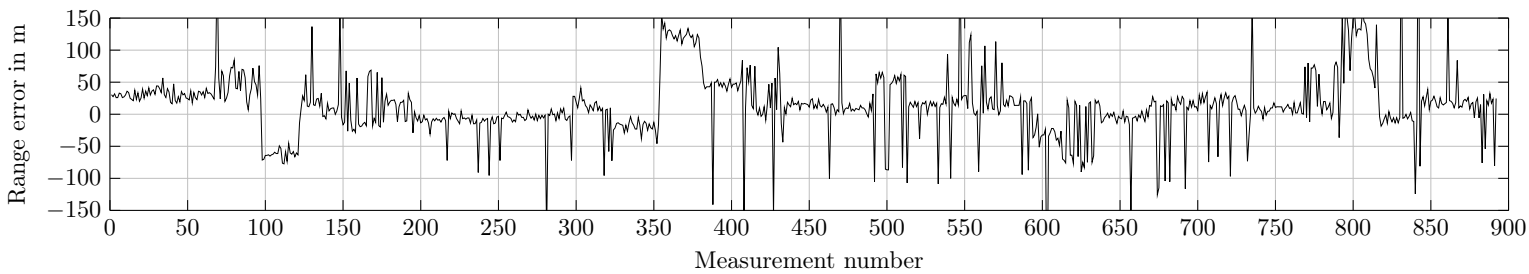

(b) Ranging error on MUSIC $\hat{d}_{\mathrm{MUSIC}}$.

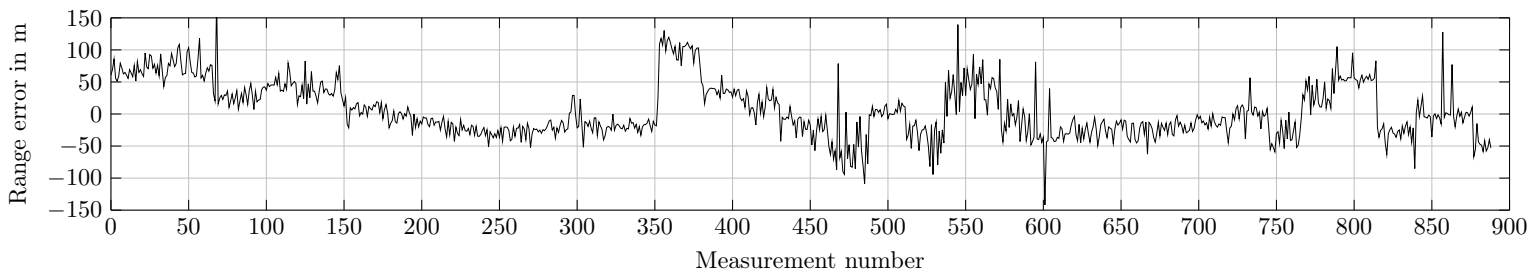

(c) Ranging error on synthetic data trained DNN $\hat{d}_{\text {S-DNN }}$.

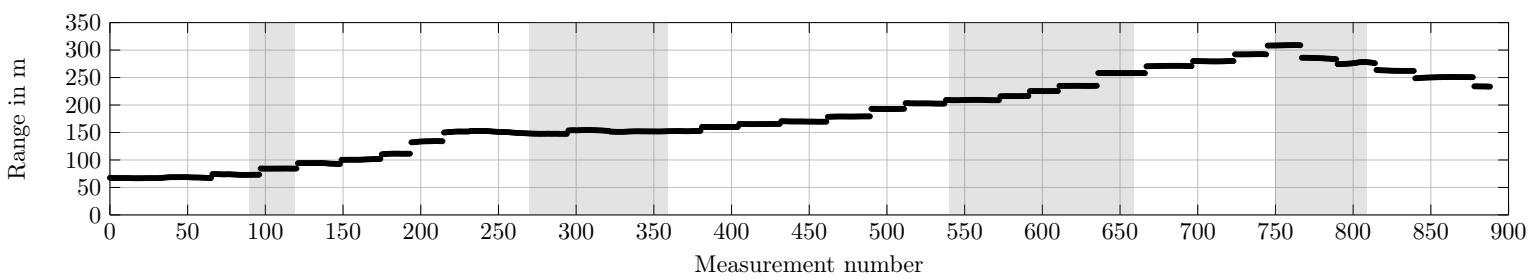

(d) GNSS ground truth (white = training data for DNN, gray = range estimation with DNN).

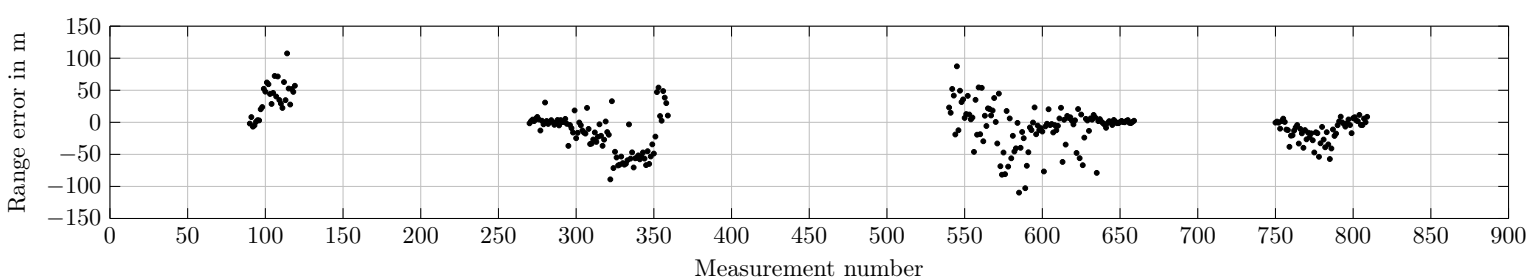

(e) Ranging error on synthetically and field trial data (mixed) trained DNN $\hat{d}_{\mathrm{M}-\mathrm{DNN}}$.

Fig. 5: Multi-channel narrowband ranging errors for the 900 field trial measurements with $10 \mathrm{kHz}$ bandwidth signals.

\section{Conclusion}

Multi-channel narrowband LPWA ranging accuracy has been evaluated by numerical simulations in a two-path propagation scenario. Parametric range estimation through MUSIC outperforms the 


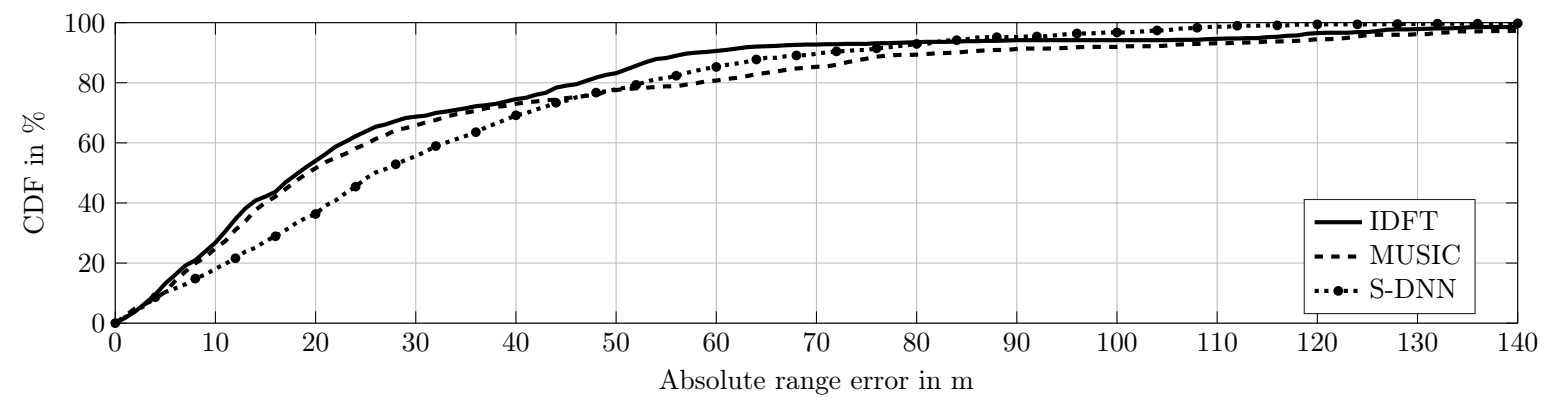

Fig. 6: Cumulative Distribution Functions (CDF) for field trial multi-channel narrowband range estimation with $10 \mathrm{kHz}$ bandwidth signals on all 900 measurements.

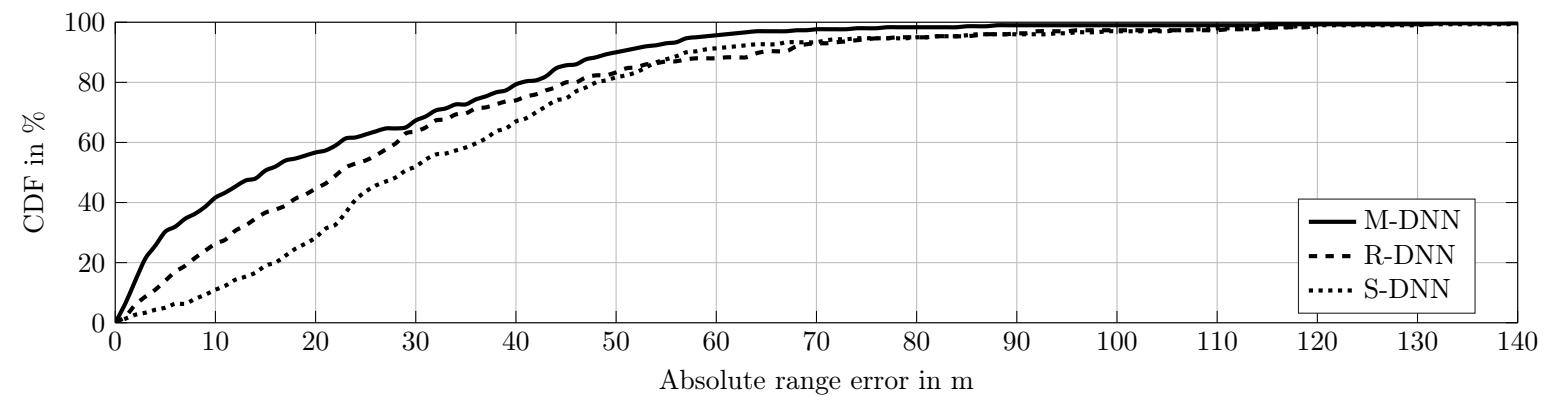

Fig. 7: CDFs for field trial multi-channel narrowband range estimation with $10 \mathrm{kHz}$ bandwidth signals on $1 / 3$ of the measurements (Fig. 5 d, gray).

state of the art Inverse Discrete Fourier Transform approach, due to its high-resolution property. For sufficient large $E_{\mathrm{s}} / N_{0}$, MUSIC achieves more accurate range estimates for close multipath than the IDFT technique in numerical simulations. Applied to real outdoor field trials, MUSIC and IDFT estimators show comparable performances (60 to $80 \mathrm{~m}$ in $90 \%$ of the cases).

The deep learning based range estimation algorithm shows high robustness to multipath, with $25 \mathrm{~m}$ error in simulation and $45 \mathrm{~m}$ in $90 \%$ of the cases on the field trial data, when trained on mixed data. Multi-channel measurements provide improved temporal resolution through sequentially increased bandwidth. Combined with deep learning techniques they are potential enablers for precise LPWA localization due to appropriated signal processing, especially for unknown and dense multipath propagation scenarios. Yet, generalization of these findings to other scenarios remains open.

Further work may consider training deep neural networks assuming more complex channel models. Extensive field trials will provide a comprehensive database for training with mixed synthetic and real data.

Future studies may investigate the combination of multi-channel ranging with beamforming strategies to mitigate multipath as well as Time-Difference-of-Arrival (TDoA) like approaches to address the complexity of the two-way signaling scheme and to ensure compatibility with the star topology of LPWA networks.

\section{References}

1. U. Raza, P. Kulkarni, and M. Sooriyabandara, "Low Power Wide Area Networks: An Overview," IEEE Communications Surveys Tutorials, vol. 19, no. 2, pp. 855-873, 2017. 
$12 / 12 \quad$ F. Wolf et al.

2. S. M. Razavi, F. Gunnarsson, H. Ryden, A. Busin, X. Lin, X. Zhang, S. Dwivedi, I. Siomina, and R. Shreevastav, "Positioning in cellular networks: Past, present, future," in 2018 IEEE Wireless Communications and Networking Conference (WCNC), April 2018, pp. 1-6.

3. Link Labs, "LoRa Localization," https://www.link-labs.com/blog/lora-localization, 2016.

4. P. Gotthard and T. Jankech, "Low-Cost Car Park Localization Using RSSI in Supervised LoRa Mesh Networks," in 2018 15th Workshop on Positioning, Navigation and Communications (WPNC), Oct 2018, pp. $1-6$.

5. M. Aernouts, B. Bellekens, R. Berkvens, and M. Weyn, "A Comparison of Signal Strength Localization Methods with Sigfox," in 2018 15th Workshop on Positioning, Navigation and Communications (WPNC), Oct 2018, pp. 1-6.

6. M. Skolnik, Radar Handbook, 2nd ed. McGrawHill, 1990.

7. N. Podevijn, D. Plets, J. Trogh, L. Martens, P. Suanet, K. Hendrikse, and W. Joseph, "TDoA-Based Outdoor Positioning with Tracking Algorithm in a Public LoRa Network," Wireless Communications and Mobile Computing, 2018.

8. F. Wolf, J.-B. Dore, X. Popon, S. de Rivaz, F. Dehmas, and J. P. Cances, "Coherent Multi-Channel Ranging for Narrowband LPWAN: Simulation and Experimentation Results," in 15th Workshop on Positioning, Navigation and Communications (WPNC), October 2018, pp. 1-6.

9. D. Vasisht, S. Kumar, and D. Katabi, "Decimeter-Level Localization with a Single WiFi Access Point," 2016.

10. M. Pichler, S. Schwarzer, A. Stelzer, and M. Vossiek, "Multi-Channel Distance Measurement With IEEE 802.15.4 (ZigBee) Devices," IEEE Journal of Selected Topics in Signal Processing, vol. 3, no. 5, pp. 845-859, Oct 2009.

11. S. Schwarzer, "Entwicklung eines industriellen Funkortungssystems basierend auf der kohaerenten Kombination von Kommunikationssignalen mit IEEE-802.15.4-Geraeten," Ph.D. dissertation, Technischen Universitaet Clausthal, 2011.

12. A. Povalac and J. Sebesta, "Phase difference of arrival distance estimation for RFID tags in frequency domain," in 2011 IEEE International Conference on RFID-Technologies and Applications, Sept 2011, pp. $188-193$.

13. W. Liao and A. Fannjiang, "MUSIC for Single-Snapshot Spectral Estimation: Stability and Super-resolution," CoRR, vol. abs/1404.1484, 2014.

14. X. Li and K. Pahlavan, "Super-resolution TOA estimation with diversity for indoor geolocation," IEEE Transactions on Wireless Communications, vol. 3, no. 1, pp. 224-234, Jan 2004.

15. A. Chehri, P. Fortier, and P. . Tardif, "On the TOA Estimation for UWB Ranging in Complex Confined Area," in 2007 International Symposium on Signals, Systems and Electronics, July 2007, pp. 533-536.

16. Q. Mao, F. Hu, and Q. Hao, "Deep Learning for Intelligent Wireless Networks: A Comprehensive Survey," IEEE Communications Surveys Tutorials, vol. 20, no. 4, pp. 2595-2621, Fourthquarter 2018.

17. O. Bialer, N. Garnett, and D. Levi, "A Deep Neural Network Approach for Time-Of- Arrival Estimation in Multipath Channels," in 2018 IEEE International Conference on Acoustics, Speech and Signal Processing (ICASSP), April 2018, pp. 2936-2940.

18. A. Niitsoo, T. Edelhäußer, E. Eberlein, N. Hadaschik, and C. Mutschler, "A Deep Learning Approach to Position Estimation from Channel Impulse Responses," Sensors, vol. 19, no. 5, p. 1064, 2019.

19. A. Zappone, M. Di Renzo, M. Debbah, T. T. Lam, and X. Qian, "Model-aided wireless artificial intelligence: Embedding expert knowledge in deep neural networks towards wireless systems optimization," arXiv preprint arXiv:1808.01672, 2018. 